\title{
SFRA, Detect Of Winding Deformation in Power Transformer
}

\author{
Shivangi Rai ${ }^{1}$, (Prof.) N.P. Gupta ${ }^{2}$ \\ ${ }^{I} M$.tech Student, EEE Department, Oriental Institute of Science \& Technology Bhopal, RGPV \\ University, M.P. India. \\ ${ }^{2}$ Professor, EEE Department, Oriental Institute of Science \& Technology Bhopal, RGPV \\ University, M.P. India.
}

\begin{abstract}
The sweep frequency response analysis is extensively used technique for detect hidden fault and condition monitoring of power transformer. The operation is carried out by supply a low voltage signal of varying frequencies to the transformer windings and measuring both the input and output signals. These two signals give the required response of the ratio is called the transfer function of the transformer from which both the magnitude and phase can be obtained. Frequency response is change as measured by SFRA techniques may indicate a physical change inside the transformer, and then causes of fault identified and investigation is required for root cause analysis.
\end{abstract}

Keywords: Sweep Frequency Response Analyzer Doble M5100, SFRA, Winding Deformation, Power Transformer, SFRA Traces

\section{Introduction}

This work proposes to detect short-circuit faults between two turns in a winding of a transformer \& mechanical displacement. The transformer is considered to be a complex network of RLC components. Any form of physical damage to the transformer results in the changes of this RLC network. These changes are what we are looking for and employ frequency response to highlight these small changes in the RLC network within the transformer. For different frequencies of the RLC network offers different impedance paths. Hence, the transfer function at each frequency is a measure of the effective impedance of the RLC network of the transformer. Any geometrical deformation changes in the RLC network, which in turn changes the transfer function at different frequencies and hence highlights the area of concern Power transformers are the most expensive and important component in a high voltage electric power system. It is one of essential elements and a failure of transformer can cause serious problems in electric utility operation. [11].

\section{Sweep Frequency Response Analyzer}

The SFRA consist of measuring the impedance of a transformer winding over a wide range of frequencies \& comparing the results with a reference set. SFRA is non-destructive test, Off-Line testing and it can be carried out for any voltage rating of Power Transformer. The measurement of SFRA can be a part of regular transformer maintenance. The SFRA Analyzer detects the following fault in the power transformer before break down occur.

1. Displacement of Core

2. Deformation and displacement of winding

3. Faulty core ground

4. Partial winding collapse

5. Hoop Buckling

6. Broken or loosened clamping structures

7. Shorted turns and open winding.

The technique of SFRA is a major advance in transformer condition analysis. This is a tested technique for obtain precise and repeatable measurements.

The test can be carried out,

1. First to obtain initial signature of the transformer sweep frequency response as a record for the future reference comparison.

2. Periodical measurement as a maintenance check, once in two years.

3. Immediately after a major external shortcuts, especially for faults electrically closer to transformer.

4. Transportation or re-location of transformer.

5. Earthquakes.

6. Pre-commissioning check.[1] 


\section{Sweep Frequency Response Test}

In the most of the commercial sweep frequency response measuring instruments, the measuring task is performed in terms of magnitude $\left(20 \times \log \left(\mathrm{V}_{2} / \mathrm{V}_{1}\right)\right)$. The meters are also capable of measuring corresponding phase and impedance magnitudes. The angle between the output voltage $V_{2}$ and the input

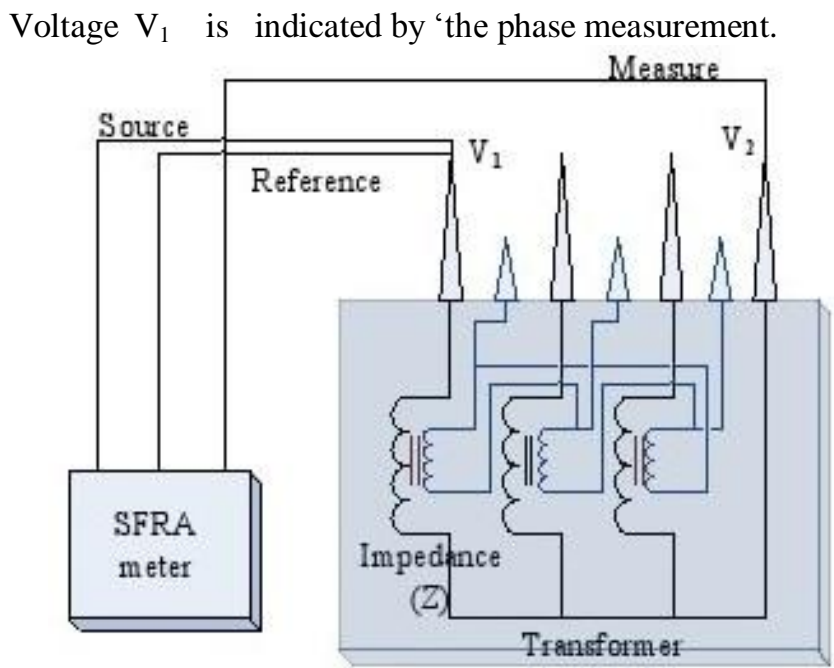

Fig. (1): A typical SFRA test connection

Commercial FR instruments have three leads i.e. source, reference and measure (Fig. 1). The source and reference terminals are connected to the input end while the measure terminal is connected to the output end. In typical three phase transformers, there are high voltage (HV), low voltage (LV) and tertiary windings, and hence numerous FR connections are possible. Major kinds of FR connections are discussed in [3,5], which include end-to-end connections and inter-winding connections. A typical pattern is observed for each test connection. Test instruments have an alternating voltage source, capable of supplying a sweep frequency in the range of $20 \mathrm{~Hz}$ to $5 \mathrm{MHz}$, with voltage magnitude between $2 \mathrm{~V}$ to $24 \mathrm{~V}$. The measurement procedures and precautions to be taken are well documented in $[6,7]$.

\section{Discussion On Typical Natures Of Sfra Plots Of Various Types Of Connection}

The end-to-end SFRA connection is one of the most commonly used connections of the SFRA test. It has two versions, i.e. end-to-end open circuit (the secondary winding kept open-circuited) and end-to-end short circuit (the secondary winding kept short-circuited). The intention of the end-to-end open circuit connection is specifically to check core health in addition to health of windings. A typical end-to-end open circuit SFRA graph for a star-connected LV winding is shown in Fig. 2. The low-frequency region (up to $10 \mathrm{kHz}$ ) has one dominant valley which is due to of resonance between the core-dominated inductance and the winding capacitance. The initial downward trend is governed by the inductance while the upward trend in the curve is dominated by the winding capacitance.

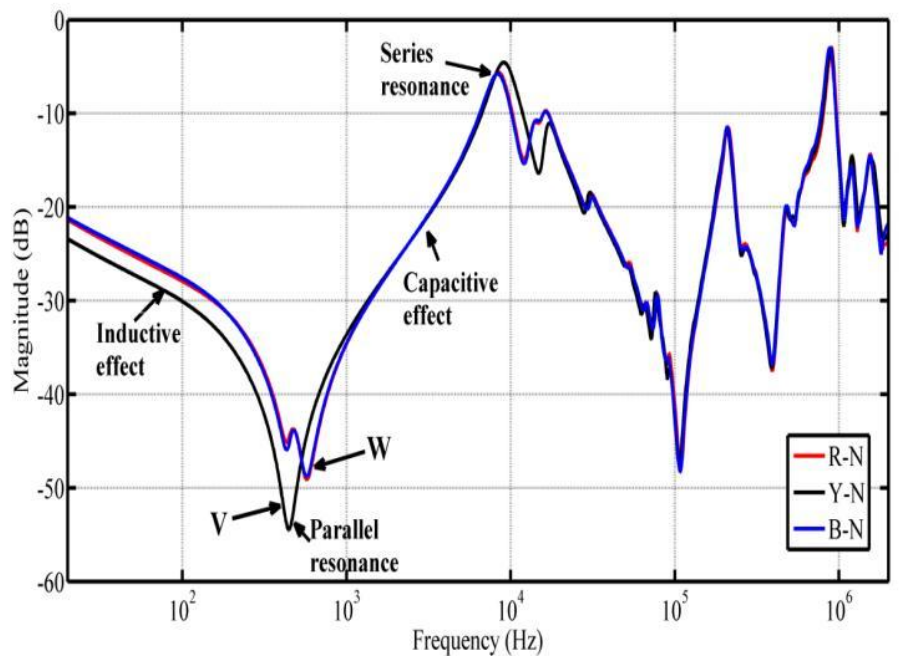

Fig. (2): End-to-end open circuit frequency response for a 10 MVA transformer 
Initially, at low frequencies the amount of flux is substantial in the core; this is responsible for higher values of the inductance and the corresponding influence of the core on the curve. As the frequency increases, the amount of flux in the core reduces for the same magnitude of the input voltage. At the resonance point the value of the inductive reactance becomes equal to that of the capacitive reactance, and thereafter the capacitive reactance becomes dominant. Thus, the position of the first valley in the case of the end-to-end open circuit SFRA connection is decided by the size of the core, the amount of excitation and the winding capacitance. The first valley point is due to parallel resonance between the core influenced inductance and the winding capacitance. In mid- and high-frequency regions the curve has many resonance points indicating series as well as parallel resonances between inductances and capacitances. Peaks are due to series resonances while valley points represent parallel resonances. A downward movement of the curve indicates an inductive behavior while an upward movement shows a capacitive behavior.

For HV and LV windings, the patterns of the SFRA curves are different. LV windings have smaller bushings (low capacitances) and lesser number of turns (low inductances) while HV windings have larger bushings (high capacitances) and more number of turns (higher inductances). This constructional difference is responsible for larger HV winding impedance compared to that of the LV winding. Thus, the LV winding's SFRA remains above that of the $\mathrm{H}$ curve. The de facto convention for the SFRA curve is: the lower the curve the higher is the impedance of the winding. In the case of the end-to-end open-circuit test condition for a starconnected $\mathrm{LV}$ winding, the curve generally starts between $-10 \mathrm{~dB}$ to $-30 \mathrm{~dB}$ and usually its movement remains between 0 to $-60 \mathrm{~dB}$. The designed impedance of the LV winding is lower than that of the HV winding in a transformer. Thus the voltage drop across the windings, while testing, is lower in the case of the LV winding. Hence, the LV winding SFRA curve has higher values of $\mathrm{dB}$ compared to the HV curve winding. The HV winding's SFRA generally ranges from $-40 \mathrm{~dB}$ to $-60 \mathrm{~dB}$ depending upon the rating and impedance of the transformer

For an end-to-end open circuit case, a star-connection involves a single winding while testing it, and hence the curve indicates a true picture of the winding as shown in Fig. 2. When the winding is connected in delta and a test signal is applied to one of the windings, the other two windings form a series connection internally and the combination comes in parallel with the winding under test. Thus the shape of the curve is different for delta-connected windings, since the test connection involves a series-parallel combination of the windings, as shown in Fig. 3. Another important observation for delta-connected windings in the low frequency region is that a dominant peak is observed (encircled). End phase responses R- and B-phases indicate slightly bumpier responses in the encircled region while the center phase (Y-phase) is observed to give a deep valley. The probable reason for the phenomenon is: the inductances and capacitances of the end phase are smaller than those of the center phase due to their constructional difference.

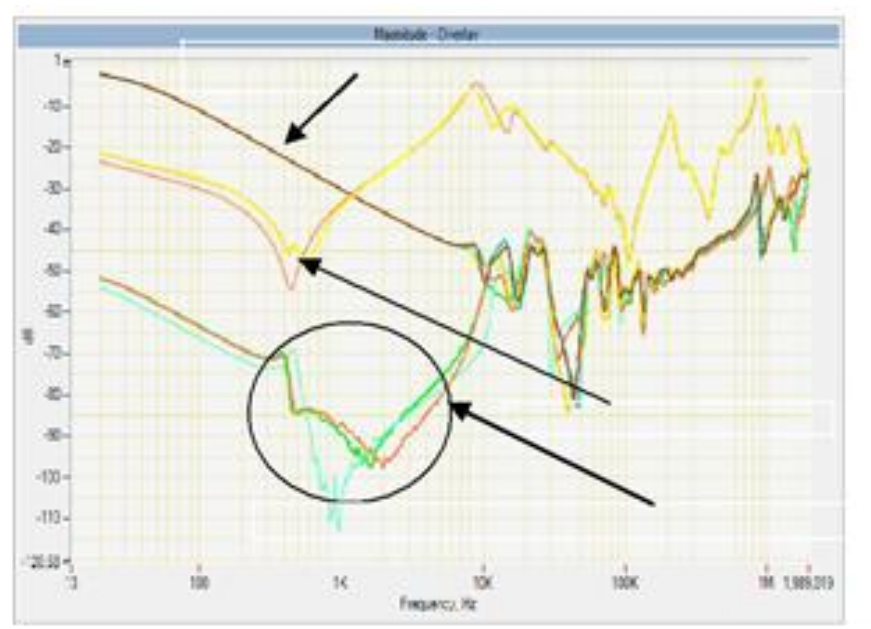

Fig. (3): SFRA of a $10 \mathrm{MVA}, 66 \mathrm{kV} / 11 \mathrm{kV}$ two winding transformer

Thus, the effective impedances for end phases remain a little lower than those of the center phase in the encircled region. This also depends on the kind of magnetism stored on each limb.

In the end-to-end short circuit connection, for a delta-connected HV winding with the LV winding short-circuited (as shown in Fig. 3), the initial valley is missing as the secondary is short-circuited, which eliminates the presence of the core for the test instrument. This connection is specifically used to check the leakage inductance along $w$ health. The mid- and high-frequency regions of the curve should be identical to those corresponding to the end-to-end open circuit connection. 


\section{Sfra Of On Auto Transformer}

Generally, very large power transformers are auto transformers. In the case of an auto transformer, the pattern of an LV to HV end-to-end FR is shown in Fig. 4, where both windings are star-connected.

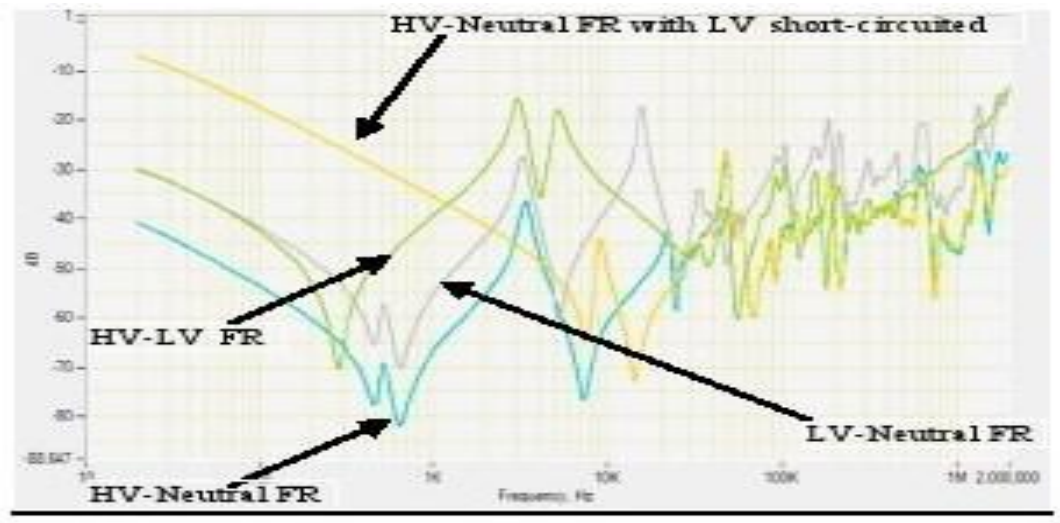

Fig. (4): SFR of B-phase for a 40 MVA auto transformer

In an auto transformer, the leakage field associated with the LV winding is lower than that of the HV winding. Its FR is a few $\mathrm{dBs}$ above than that of the $\mathrm{HV}$ winding, and has a similar shape. The end-to-end FR of the HV winding, with the LV winding kept short-circuited will have no first dominant valley because of presence the short-circuited winding. The HV-LV FR has a very different shape compared to that of a twowinding power transformer, as this connection takes the FR of the series HV winding of the auto transformer, which is not common between them, and hence the FR contains a first valley influenced by the core.

\section{Discuss What If The Curve Is Deviating From Its Typical Shape?}

A practicing engineer, who has an expertise in the measurement of FR curves, normally expects typical shapes depending upon the type of the test connection. In the event of any abnormality or fault, the curve will deviate significantly from its typical shape. Under such circumstances, there is no need to compare it with its fingerprint curve, and the abnormal shape is good enough to suspect abnormality. Fig. 5 shows such a case which is a frequency response curve for a $5 \mathrm{MVA}, 66 \mathrm{kV} / 11.55 \mathrm{kV}$ transformer for an LV end-to-end open circuit connection. The shape is appreciably different from the one in Fig. 2. Thus, this is a clear case of abnormality. When the transformer was opened, a fault (short-circuit) was observed between the LV winding and the core. As explained in a previous section, a resistance creates a damping effect and tries to flatten the curve. The flat responses for two of the phases indicate the involvement of some resistance (may be ground resistance) in the circuit, which has forced the curve to be flat for the low-frequency region. In the mid-and high-frequency regions the curve has shown typical normal patterns

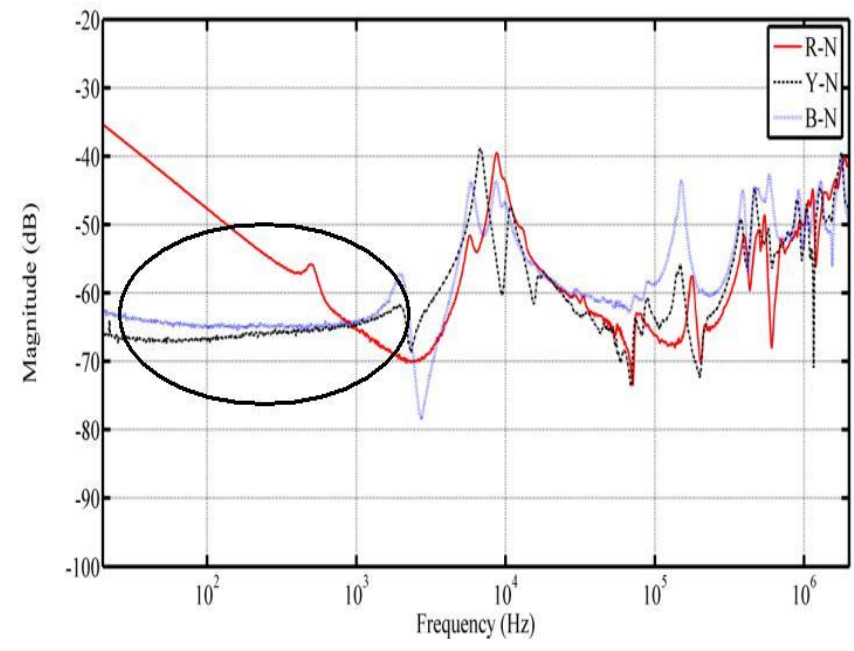

Fig. (5): SFR for a 5 MVA transformer with a fault condition 


\section{Sequence Of Test Procedure}

Practicing engineers; who often do FR measurements, very well know the effect of stored magnetism in the core in the low-frequency region. The switching-off instant for a transformer cannot be easily controlled and hence the amount of stored magnetism in the core may have different values at different times. Ideally, the SFRA measurement should be done by allowing the residual magnetism to decay down to a negligible value. This may require a long time, which may not be acceptable in practice. Another alternative is to remove the residual flux by some kind of short-circuit test, e.g. if the end-to-end short circuit test is done first, it will wipe out the flux from the core and the next FRA tests would be free from the effect of stored magnetism. Secondly, while doing a number of maintenance tests like turns ratio tests, low voltage open circuit and short circuit tests, magnetic balance tests, and M5100 tests, the SFRA test should be done just after the alternating voltage tests. Tests like the M5100 test and the winding resistance test, which invariably require a DC supply, may result in DC magnetization of the core, which may affect the low-frequency region of the FR curve.

By comparing future traces with baseline traces, the following can be noted.[8]

\begin{tabular}{|l|l|}
\hline Comparison Of Future Traces With Base Line Traces & Nature Of Problem /Fault \\
\hline $\begin{array}{l}\text { The Traces Will Change Shape And Be Distorted In The Low } \\
\text { Frequency Range (Under 5,000 Hz) }\end{array}$ & Core Problem \\
\hline $\begin{array}{l}\text { The Traces Will Be Distorted And Change Shape In Higher } \\
\text { Frequencies (Above10,000 Hz) }\end{array}$ & Winding Problem \\
\hline Changes Of Less Than 3decibels (Db) Compared To Baseline Traces. & Normal And Within Tolerances. \\
\hline From 5 Hz To $2 \mathrm{Khz}$ Changes Of +/-3 Db (Or More) & $\begin{array}{l}\text { Shorted Turns, Open Circuit, Residual } \\
\text { Magnetism, Or Core Movement. }\end{array}$ \\
\hline From 50 Hz To 20 Khz Changes Of +/- $3 \mathrm{Db}($ Or More) & $\begin{array}{l}\text { Bulk Movement Of Windings Relative } \\
\text { To Each Other. }\end{array}$ \\
\hline From 500 Hz To 2 Mhz Changes Of +/-3 Db (Or More) & $\begin{array}{l}\text { Deformation Within A Winding. } \\
\text { From } 25 \mathrm{~Hz} \text { To } 10 \mathrm{Mhz} \text { Changes Of +/-3 Db (Or More) }\end{array}$ \\
\hline
\end{tabular}

\section{Conclusion}

This paper has outlined the basic theory of SFRA. Basic circuit diagram for test connection are explained the typical shape of end to end SFRA connections for an open as well as a short circuited condition, SFRA trends in HP and LV windings and in Star-Delta windings and the nature of the SFRA in auto transformer are discussed. These discussions are expected to help practicing engineers in doing better diagnostics.

\section{References}

[1] Sweep Frequency Response Analysis (SFRA) A Major Advance in Transformer Condition Analysis. Power Linker Group

[2] A. Kraetge, M.Kruger, J.L.Volaasquez (Omicron electronics GmbH, Austria), H.Viljoen, A.Dierks, (Aleetrix (Pty) Ltd., South Africa), Aspects of the Practical Application of Sweep Frequency Response Analysis (SFRA) on Power Transformers), Cigre $20096^{\text {th }}$ Southern Africa Regional Conference.

[3] Cigre, "Mechanical condition assessment of transformer windings using FRA (Frequency Response Analysis)" Cigre working Group A2.26, April 2008 .

[4] G. M. Kennedy, A. J. McGrailland J.A. Lapworth. (October 2007) "Transformer sweep frequency response analysis (SFRA)". Energize. Pp.28-33

[5] IEEE-SFRA, "IEEE guide for the application and interpretation of frequency response analysis of oil-immersed transformers," IEEE Std C57. 149-2012, March 2013.

[6] C. Sweetser and T. McGrail, "Sweep frequency response analysis transformer applications," presented at the Relatoriotrcnico, Doble Engineering Company, 2003.

[7] C. Bergman. SFRA- "An application that creates customer value?" [Online].Available: www.paxdiagnostics.com/uploads/Thesis EXA468 SFRA r4.pdf

[8] Facilities Instructions, Standards and Techniques Volume 3-31 Transformer Diagnostics June 2003 United States Department of the Interior Bureau of Reclamation

[9] Yog Raj Sood, Rajkumar Jarial, Kapli Gandhi, Condition Monitoring of Power Transformer Using Sweep Frequency Response Analysis, MIT International Journal of Electrical and Instrumentation Engineering Vol 1, No.2, Aug. 2011, pp 80-86.

[10] Feature Transformer Frequency Response Analysis: Introduction Spring 2005.

[11] Luwendran Moodley, Brian de Klerk, Sweep Frequency Response Analysis as a Diagnostic Tool to Detect Transformer Mechanical Integrity.

[12] Charles Sweester, Dr. Tony McGrail, Sweep Frequency Response Analysis Transformer Applications, A Technical Paper from Doble Engineering. 